\title{
Solvability of Some Boundary Value Problems for Fractional $p$-Laplacian Equation
}

\author{
Taiyong Chen and Wenbin Liu \\ Department of Mathematics, China University of Mining and Technology, Xuzhou 221116, China \\ Correspondence should be addressed to Taiyong Chen; taiyongchen@cumt.edu.cn
}

Received 4 July 2013; Accepted 9 September 2013

Academic Editor: Chuanzhi Bai

Copyright (C) 2013 T. Chen and W. Liu. This is an open access article distributed under the Creative Commons Attribution License, which permits unrestricted use, distribution, and reproduction in any medium, provided the original work is properly cited.

This paper considers the existence of solutions for two boundary value problems for fractional $p$-Laplacian equation. Under certain nonlinear growth conditions of the nonlinearity, two new existence results are obtained by using Schaefer's fixed point theorem. As an application, an example to illustrate our results is given.

\section{Introduction}

Fractional calculus is a generalization of ordinary differentiation and integration on an arbitrary order that can be noninteger. This subject, as old as the problem of ordinary differential calculus, can go back to the times when Leibniz and Newton invented differential calculus. As is known to all, the problem for fractional derivative was originally raised by Leibniz in a letter, dated September 30, 1695. A fractional derivative arises from many physical processes, such as a non-Markovian diffusion process with memory [1], charge transport in amorphous semiconductors [2], and propagations of mechanical waves in viscoelastic media [3], and so forth. Moreover, phenomena in electromagnetics, acoustics, viscoelasticity, electrochemistry, and material science are also described by differential equations of fractional order [48]. For instance, Pereira et al. [9] considered the following fractional Van der Pol equation:

$$
D^{\lambda} x(t)+\alpha\left(x^{2}(t)-1\right) x^{\prime}(t)+x(t)=0, \quad 1<\lambda<2,
$$

where $D^{\lambda}$ is the fractional derivative of order $\lambda$ and $\alpha$ is a control parameter that reflects the degree of nonlinearity of the system. Equation (1) is obtained by substituting the capacitance by a fractance in the nonlinear RLC circuit model.

Recently, fractional differential equations have been of great interest due to the intensive development of the theory of fractional calculus itself and its applications. For example, for fractional initial value problems, the existence and multiplicity of solutions (or positive solutions) were discussed in [10-13]. On the other hand, for fractional boundary value problems (FBVPs), Agarwal et al. [14] considered a twopoint boundary value problem at nonresonance, and Bai [15] considered a $m$-point boundary value problem at resonance. For more papers on FBVPs, see [16-21] and the references therein.

The turbulent flow in a porous medium is a fundamental mechanics problem. For studying this type of problems, Leibenson [22] introduced the $p$-Laplacian equation as follows:

$$
\left(\phi_{p}\left(x^{\prime}(t)\right)\right)^{\prime}=f\left(t, x(t), x^{\prime}(t)\right)
$$

where $\phi_{p}(s)=|s|^{p-2} s, p>1$. Obviously, $\phi_{p}$ is invertible and its inverse operator is $\phi_{q}$, where $q>1$ is a constant such that $1 / p+1 / q=1$

In the past few decades, many important results relative to (2) with certain boundary value conditions have been obtained. We refer the reader to [23-27] and the references cited therein. For boundary value problems of fractional $p$-Laplacian equations, Chen and Liu [28] considered an antiperiodic boundary value problem with the following form:

$$
\begin{aligned}
& D_{0^{+}}^{\beta} \phi_{p}\left(D_{0^{+}}^{\alpha} x(t)\right)=f(t, x(t)), \quad t \in[0,1], \\
& x(0)=-x(1), \quad D_{0^{+}}^{\alpha} x(0)=-D_{0^{+}}^{\alpha} x(1),
\end{aligned}
$$


where $0<\alpha, \beta \leq 1,1<\alpha+\beta \leq 2$, and $D_{0^{+}}^{\alpha}$ is Caputo fractional derivative. Under certain nonlinear growth conditions of the nonlinearity, an existence result was obtained by using degree theory. In addition, Yao et al. [29] studied a three-point boundary value problem given by

$$
\begin{gathered}
-D_{t}^{\beta}\left(\phi_{p}\left(D_{t}^{\alpha} x\right)\right)(t)=h(t) f(t, x(t)), \quad t \in(0,1), \\
x(0)=0, \quad D_{t}^{\gamma} x(1)=a D_{t}^{\gamma} x(\xi), \quad D_{t}^{\alpha} x(0)=0,
\end{gathered}
$$

where $D_{t}^{\alpha}$ is the standard Riemann-Liouville derivative with $1<\alpha \leq 2,0<\beta, \gamma \leq 1,0 \leq \alpha-\gamma-1, \xi \in(0,1)$, and the constant $a$ is a positive number satisfying $a \xi^{\alpha-\gamma-2} \leq 1-\gamma$. The monotone iterative technique was applied to establish the existence results on multiple positive solutions in [29].

Motivated by the works mentioned previously, in this paper, we investigate the existence of solutions for fractional $p$-Laplacian equation of the form

$$
D_{0^{+}}^{\beta} \phi_{p}\left(D_{0^{+}}^{\alpha} x(t)\right)=f\left(t, x(t), D_{0^{+}}^{\alpha} x(t)\right), \quad t \in[0,1]
$$

subject to either boundary value conditions

$$
x(0)=D_{0^{+}}^{\alpha} x(1)=0
$$

or

$$
x(0)=\int_{0}^{1} D_{0^{+}}^{\alpha} x(t) d t=0,
$$

where $0<\alpha, \beta \leq 1,1<\alpha+\beta \leq 2, D_{0^{+}}^{\alpha}$ is a Caputo fractional derivative, and $f:[0,1] \times \mathbb{R}^{2} \rightarrow \mathbb{R}$ is continuous.

Note that the nonlinear operator $D_{0^{+}}^{\beta} \phi_{p}\left(D_{0^{+}}^{\alpha}\right)$ reduces to the linear operator $D_{0^{+}}^{\beta} D_{0^{+}}^{\alpha}$ when $p=2$ and the additive index law

$$
D_{0^{+}}^{\beta} D_{0^{+}}^{\alpha} u(t)=D_{0^{+}}^{\alpha+\beta} u(t)
$$

holds under some reasonable constraints on the function $u(t)$ [30].

The rest of this paper is organized as follows. Section 2 contains some necessary notations, definitions, and lemmas. In Section 3, based on Schaefer's fixed point theorem, we establish two theorems on existence of solutions for FBVP (5) and (6) (Theorem 7) and FBVP (5) and (7) (Theorem 8). Finally, in Section 4, an explicit example is given to illustrate the main results. Our results are different from those of bibliographies listed in the previous texts.

\section{Preliminaries}

For the convenience of the reader, we present here some necessary basic knowledge and definitions about fractional calculus theory, which can be found, for instance, in $[31,32]$.

Definition 1. The Riemann-Liouville fractional integral operator of order $\alpha>0$ of a function $u:(0,+\infty) \rightarrow \mathbb{R}$ is given by

$$
I_{0^{+}}^{\alpha} u(t)=\frac{1}{\Gamma(\alpha)} \int_{0}^{t}(t-s)^{\alpha-1} u(s) d s,
$$

provided that the right side integral is pointwise defined on $(0,+\infty)$.

Definition 2. The Caputo fractional derivative of order $\alpha>0$ of a continuous function $u:(0,+\infty) \rightarrow \mathbb{R}$ is given by

$$
\begin{aligned}
D_{0^{+}}^{\alpha} u(t) & =I_{0^{+}}^{n-\alpha} \frac{d^{n} u(t)}{d t^{n}} \\
& =\frac{1}{\Gamma(n-\alpha)} \int_{0}^{t}(t-s)^{n-\alpha-1} u^{(n)}(s) d s,
\end{aligned}
$$

where $n$ is the smallest integer greater than or equal to $\alpha$, provided that the right side integral is pointwise defined on $(0,+\infty)$.

Lemma 3 (see [33]). Let $\alpha>0$. Assume that $u, D_{0^{+}}^{\alpha} u \in L(0,1)$. Then the following equality holds:

$$
I_{0^{+}}^{\alpha} D_{0^{+}}^{\alpha} u(t)=u(t)+c_{0}+c_{1} t+\cdots+c_{n-1} t^{n-1},
$$

where $c_{i} \in \mathbb{R}, i=0,1, \ldots, n-1$; here $n$ is the smallest integer greater than or equal to $\alpha$.

Lemma 4 (see $[34]$ ). For fixed $l \in C[0,1]$, let one define

$$
G_{l}(a)=\int_{0}^{1} \phi_{q}(l(t)+a) d t .
$$

Then the equation $G_{l}(a)=0$ has a unique solution $\tilde{a}(l)$.

In this paper, we take $Y=C[0,1]$ with the norm $\|y\|_{\infty}=$ $\max _{t \in[0,1]}|y(t)|$ and $X=\left\{x \mid x, D_{0^{+}}^{\alpha} x \in Y\right\}$ with the norm $\|x\|_{X}=\max \left\{\|x\|_{\infty},\left\|D_{0^{+}}^{\alpha} x\right\|_{\infty}\right\}$. By means of the linear functional analysis theory, we can prove that $X$ is a Banach space.

\section{Existence Results}

In this section, two theorems on existence of solutions for FBVP (5) and (6) and FBVP (5) and (7) will be given under nonlinear growth restriction of $f$.

As a consequence of Lemma 3, we have the following results that are useful in what follows.

Lemma 5. Given $h \in Y$, the unique solution of

$$
\begin{gathered}
D_{0^{+}}^{\beta} \phi_{p}\left(D_{0^{+}}^{\alpha} x(t)\right)=h(t), \quad t \in[0,1], \\
x(0)=D_{0^{+}}^{\alpha} x(1)=0
\end{gathered}
$$

is

$$
\begin{gathered}
x(t)=I_{0^{+}}^{\alpha} \phi_{q}\left(I_{0^{+}}^{\beta} h(t)+A h(t)\right) \\
=\frac{1}{\Gamma(\alpha)} \int_{0}^{t}(t-s)^{\alpha-1} \phi_{q}\left(\frac{1}{\Gamma(\beta)} \int_{0}^{s}(s-\tau)^{\beta-1} h(\tau) d \tau\right. \\
+A h(s)) d s,
\end{gathered}
$$


where

$$
\begin{aligned}
A h(t) & =-\left.I_{0^{+}}^{\beta} h(t)\right|_{t=1} \\
& =-\frac{1}{\Gamma(\beta)} \int_{0}^{1}(1-s)^{\beta-1} h(s) d s .
\end{aligned}
$$

Proof. Assume that $x(t)$ satisfies the equation of FBVP (13); then Lemma 3 implies that

$$
\phi_{p}\left(D_{0^{+}}^{\alpha} x(t)\right)=I_{0^{+}}^{\beta} h(t)+c_{0}, \quad c_{0} \in \mathbb{R} .
$$

From the boundary value condition $D_{0^{+}}^{\alpha} x(1)=0$, one has

$$
c_{0}=-\left.I_{0^{+}}^{\beta} h(t)\right|_{t=1}=A h(t) .
$$

Thus, we have

$$
x(t)=I_{0^{+}}^{\alpha} \phi_{q}\left(I_{0^{+}}^{\beta} h(t)+A h(t)\right)+c_{1}, \quad c_{1} \in \mathbb{R} .
$$

By condition $x(0)=0$, we get $c_{1}=0$. The proof is complete.

Define the operator $F: X \rightarrow X$ by

$$
\begin{aligned}
& F x(t) \\
& =I_{0^{+}}^{\alpha} \phi_{q}\left(I_{0^{+}}^{\beta} N x(t)+A N x(t)\right) \\
& =\frac{1}{\Gamma(\alpha)} \int_{0}^{t}(t-s)^{\alpha-1} \\
& \times \phi_{q}\left(\frac{1}{\Gamma(\beta)} \int_{0}^{s}(s-\tau)^{\beta-1}\right. \\
& \quad \times f\left(\tau, x(\tau), D_{0^{+}}^{\alpha} x(\tau)\right) d \tau \\
& -\frac{1}{\Gamma(\beta)} \int_{0}^{1}(1-\tau)^{\beta-1} \\
& \left.\times f\left(\tau, x(\tau), D_{0^{+}}^{\alpha} x(\tau)\right) d \tau\right) d s, \\
& \quad \forall t \in[0,1],
\end{aligned}
$$

where $N: X \rightarrow Y$ is the Nemytskii operator defined by

$$
N x(t)=f\left(t, x(t), D_{0^{+}}^{\alpha} x(t)\right), \quad \forall t \in[0,1] .
$$

Clearly, the fixed points of the operator $F$ are solutions of FBVP (5) and (6).

Lemma 6. Given $h \in Y$, the unique solution of

$$
\begin{gathered}
D_{0^{+}}^{\beta} \phi_{p}\left(D_{0^{+}}^{\alpha} x(t)\right)=h(t), \quad t \in[0,1], \\
x(0)=\int_{0}^{1} D_{0^{+}}^{\alpha} x(t) d t=0
\end{gathered}
$$

is

$$
\begin{gathered}
x(t)=I_{0^{+}}^{\alpha} \phi_{q}\left(I_{0^{+}}^{\beta} h(t)+B h(t)\right) \\
=\frac{1}{\Gamma(\alpha)} \int_{0}^{t}(t-s)^{\alpha-1} \phi_{q}\left(\frac{1}{\Gamma(\beta)} \int_{0}^{s}(s-\tau)^{\beta-1} h(\tau) d \tau\right. \\
+B h(s)) d s,
\end{gathered}
$$

where

$$
\begin{aligned}
B h(t) & =-\left.I_{0^{+}}^{\beta} h(t)\right|_{t=\eta(h)} \\
& =-\frac{1}{\Gamma(\beta)} \int_{0}^{\eta(h)}(\eta(h)-s)^{\beta-1} h(s) d s,
\end{aligned}
$$

here $\eta(h) \in(0,1)$ is a constant dependent on $h(t)$.

Proof. Assume that $x(t)$ satisfies the equation of FBVP (21); then Lemma 3 implies that

$$
D_{0^{+}}^{\alpha} x(t)=\phi_{q}\left(I_{0^{+}}^{\beta} h(t)+c_{0}\right), \quad c_{0} \in \mathbb{R} .
$$

From condition $\int_{0}^{1} D_{0^{+}}^{\alpha} x(t) d t=0$, one has

$$
\int_{0}^{1} \phi_{q}\left(I_{0^{+}}^{\beta} h(t)+c_{0}\right) d t=0 .
$$

Based on Lemma 4, we know that (25) has a unique solution $\widetilde{c_{0}}(h)$. Moreover, by the integral mean value theorem, there exists a constant $\eta(h) \in(0,1)$ such that $\phi_{q}\left(I_{0^{+}}^{\beta} h(t)+\right.$ $\left.\widetilde{c_{0}}(h)\right)\left.\right|_{t=\eta(h)}=0$, which implies that $\left.\left(I_{0^{+}}^{\beta} h(t)+\widetilde{c_{0}}(h)\right)\right|_{t=\eta(h)}=0$. Thus, we have

$$
\widetilde{c_{0}}(h)=-\left.I_{0^{+}}^{\beta} h(t)\right|_{t=\eta(h)}=B h(t) .
$$

Hence

$$
x(t)=I_{0^{+}}^{\alpha} \phi_{q}\left(I_{0^{+}}^{\beta} h(t)+B h(t)\right)+c_{1}, \quad c_{1} \in \mathbb{R} .
$$

From condition $x(0)=0$, we get $c_{1}=0$. The proof is complete. 
Define the operator $P: X \rightarrow X$ by

$P x(t)$

$$
\begin{aligned}
& =I_{0^{+}}^{\alpha} \phi_{q}\left(I_{0^{+}}^{\beta} N x(t)+B N x(t)\right) \\
& =\frac{1}{\Gamma(\alpha)} \int_{0}^{t}(t-s)^{\alpha-1} \\
& \quad \times \phi_{q}\left(\frac{1}{\Gamma(\beta)} \int_{0}^{s}(s-\tau)^{\beta-1}\right. \\
& \quad \times f\left(\tau, x(\tau), D_{0^{+}}^{\alpha} x(\tau)\right) d \tau \\
& \quad-\frac{1}{\Gamma(\beta)} \int_{0}^{\eta(x)}(\eta(x)-\tau)^{\beta-1} \\
& \left.\quad \times f\left(\tau, x(\tau), D_{0^{+}}^{\alpha} x(\tau)\right) d \tau\right) d s,
\end{aligned}
$$$$
\forall t \in[0,1] \text {, }
$$

where $\eta(x) \in(0,1)$ and $N$ is the Nemytskii operator defined by (20). Clearly, the fixed points of the operator $P$ are solutions of FBVP (5) and (7).

Our first result, based on Schaefer's fixed point theorem and Lemma 5, is stated as follows.

Theorem 7. Let $f:[0,1] \times \mathbb{R}^{2} \rightarrow \mathbb{R}$ be continuous. Assume that

$(H)$ there exist nonnegative functions $a, b, c \in Y$ such that

$$
\begin{aligned}
|f(t, u, v)| \leq & a(t)+b(t)|u|^{p-1} \\
& +c(t)|v|^{p-1}, \quad \forall t \in[0,1],(u, v) \in \mathbb{R}^{2} .
\end{aligned}
$$

Then FBVP (5) and (6) has at least one solution, provided that

$$
\frac{2}{\Gamma(\beta+1)}\left(\frac{\|b\|_{\infty}}{(\Gamma(\alpha+1))^{p-1}}+\|c\|_{\infty}\right)<1
$$

Proof. We will use Schaefer's fixed point theorem to prove that $F$ has a fixed point. The proof will be given in the following two steps.

Step 1.F : $X \rightarrow X$ is completely continuous.

Let $\Omega \subset X$ be an open bounded subset. By the continuity of $f$, we can get that $F$ is continuous and $F(\bar{\Omega})$ is bounded. Moreover, there exists a constant $T>0$ such that $\mid I_{0^{+}}^{\beta} N x+$ $A N x \mid \leq T$, for all $x \in \bar{\Omega}, t \in[0,1]$. Thus, in view of the Arzelà-Ascoli theorem, we need only to prove that $F(\bar{\Omega}) \subset X$ is equicontinuous.
For $0 \leq t_{1}<t_{2} \leq 1$ and $x \in \bar{\Omega}$, we have

$$
\begin{aligned}
& \left|F x\left(t_{2}\right)-F x\left(t_{1}\right)\right| \\
& =\frac{1}{\Gamma(\alpha)} \mid \int_{0}^{t_{2}}\left(t_{2}-s\right)^{\alpha-1} \phi_{q}\left(I_{0^{+}}^{\beta} N x(s)+A N x(s)\right) d s \\
& -\int_{0}^{t_{1}}\left(t_{1}-s\right)^{\alpha-1} \phi_{q}\left(I_{0^{+}}^{\beta} N x(s)+A N x(s)\right) d s \\
& \leq \frac{T^{q-1}}{\Gamma(\alpha)}\left\{\int_{0}^{t_{1}}\left[\left(t_{1}-s\right)^{\alpha-1}-\left(t_{2}-s\right)^{\alpha-1}\right] d s\right. \\
& \left.+\int_{t_{1}}^{t_{2}}\left(t_{2}-s\right)^{\alpha-1} d s\right\} \\
& =\frac{T^{q-1}}{\Gamma(\alpha+1)}\left[t_{1}^{\alpha}-t_{2}^{\alpha}+2\left(t_{2}-t_{1}\right)^{\alpha}\right] \\
& \leq \frac{T^{q-1}}{\Gamma(\alpha+1)}\left[t_{2}^{\alpha}-t_{1}^{\alpha}+2\left(t_{2}-t_{1}\right)^{\alpha}\right] .
\end{aligned}
$$

Since $t^{\alpha}$ is uniformly continuous on $[0,1]$, we can obtain that $F(\bar{\Omega}) \subset Y$ is equicontinuous. A similar proof can show that $\left(I_{0^{+}}^{\beta} N+A N\right)(\bar{\Omega}) \subset Y$ is equicontinuous. This, together with the uniform continuity of $\phi_{q}(s)$ on $[-T, T]$, yields that $D_{0^{+}}^{\alpha} F(\bar{\Omega})\left(=\phi_{q}\left(I_{0^{+}}^{\beta} N+A N\right)(\bar{\Omega})\right) \subset Y$ is also equicontinuous.

Step 2 (priori bounds). Set

$$
\Omega=\left\{x \in X \mid x=\lambda^{q-1} F x, \lambda \in(0,1)\right\} .
$$

Now it remains to show that the set $\Omega$ is bounded.

From Lemma 3 and boundary value condition $x(0)=0$, one has

$$
\begin{aligned}
x(t) & =I_{0+}^{\alpha} D_{0^{+}}^{\alpha} x(t) \\
& =\frac{1}{\Gamma(\alpha)} \int_{0}^{t}(t-s)^{\alpha-1} D_{0^{+}}^{\alpha} x(s) d s .
\end{aligned}
$$

Thus, we get

$$
\begin{aligned}
|x(t)| & \leq \frac{1}{\Gamma(\alpha)} \int_{0}^{t}(t-s)^{\alpha-1}\left|D_{0^{+}}^{\alpha} x(s)\right| d s \\
& \leq \frac{1}{\Gamma(\alpha)}\left\|D_{0^{+}}^{\alpha} x\right\|_{\infty} \cdot \frac{1}{\alpha} t^{\alpha} \\
& \leq \frac{1}{\Gamma(\alpha+1)}\left\|D_{0^{+}}^{\alpha} x\right\|_{\infty}, \quad \forall t \in[0,1] .
\end{aligned}
$$

That is,

$$
\|x\|_{\infty} \leq \frac{1}{\Gamma(\alpha+1)}\left\|D_{0^{+}}^{\alpha} x\right\|_{\infty} .
$$


For $x \in \Omega$, we have

$$
\begin{aligned}
\phi_{p}\left(D_{0^{+}}^{\alpha} x(t)\right)= & \lambda\left(I_{0^{+}}^{\beta} N x(t)+A N x(t)\right) \\
= & \frac{\lambda}{\Gamma(\beta)} \int_{0}^{t}(t-s)^{\beta-1} f\left(s, x(s), D_{0^{+}}^{\alpha} x(s)\right) d s \\
& -\frac{\lambda}{\Gamma(\beta)} \int_{0}^{1}(1-s)^{\beta-1} f\left(s, x(s), D_{0^{+}}^{\alpha} x(s)\right) d s .
\end{aligned}
$$

So, from $(H)$, we obtain that

$$
\begin{aligned}
& \left|\phi_{p}\left(D_{0^{+}}^{\alpha} x(t)\right)\right| \\
& \leq \frac{2}{\Gamma(\beta)} \int_{0}^{1}(1-s)^{\beta-1}\left|f\left(s, x(s), D_{0^{+}}^{\alpha} x(s)\right)\right| d s \\
& \leq \frac{2}{\Gamma(\beta)} \int_{0}^{1}(1-s)^{\beta-1} \\
& \times\left(a(s)+b(s)|x(s)|^{p-1}+c(s)\left|D_{0^{+}}^{\alpha} x(s)\right|^{p-1}\right) d s \\
& \leq \frac{2}{\Gamma(\beta)}\left(\|a\|_{\infty}+\|b\|_{\infty}\|x\|_{\infty}^{p-1}+\|c\|_{\infty}\left\|D_{0^{+}}^{\alpha} x\right\|_{\infty}^{p-1}\right) \cdot \frac{1}{\beta} \\
& =\frac{2}{\Gamma(\beta+1)}\left(\|a\|_{\infty}+\|b\|_{\infty}\|x\|_{\infty}^{p-1}+\|c\|_{\infty}\left\|D_{0^{+}}^{\alpha} x\right\|_{\infty}^{p-1}\right),
\end{aligned}
$$$$
\forall t \in[0,1],
$$

which, together with $\left|\phi_{p}\left(D_{0^{+}}^{\alpha} x(t)\right)\right|=\left|D_{0^{+}}^{\alpha} x(t)\right|^{p-1}$ and (35), yields that

$$
\begin{aligned}
\left\|D_{0^{+}}^{\alpha} x\right\|_{\infty}^{p-1} \leq & \frac{2}{\Gamma(\beta+1)} \\
& \times\left[\|a\|_{\infty}+\frac{\|b\|_{\infty}}{(\Gamma(\alpha+1))^{p-1}}\left\|D_{0^{+}}^{\alpha} x\right\|_{\infty}^{p-1}\right. \\
& \left.+\|c\|_{\infty}\left\|D_{0^{+}}^{\alpha} x\right\|_{\infty}^{p-1}\right] .
\end{aligned}
$$

In view of (30), from (38), we can see that there exists a constant $M_{1}>0$ such that

$$
\left\|D_{0^{+}}^{\alpha} x\right\|_{\infty} \leq M_{1}
$$

Thus, from (35), we get

$$
\|x\|_{\infty} \leq \frac{M_{1}}{\Gamma(\alpha+1)}:=M_{2} .
$$

Combining (39) with (40), we have

$$
\|x\|_{X}=\max \left\{\|x\|_{\infty},\left\|D_{0^{+}}^{\alpha} x\right\|_{\infty}\right\} \leq \max \left\{M_{1}, M_{2}\right\} .
$$

As a consequence of Schaefer's fixed point theorem, we deduce that $F$ has a fixed point which is the solution of FBVP (5) and (6). The proof is complete.
Our second result, based on Schaefer's fixed point theorem and Lemma 6, is stated as follows.

Theorem 8. Let $f:[0,1] \times \mathbb{R}^{2} \rightarrow \mathbb{R}$ be continuous. Suppose that $(H)$ holds; then FBVP (5) and (7) has at least one solution, provided that (30) is satisfied.

Proof. The proof work is similar to the proof of Theorem 7, so we omit the details.

\section{An Example}

In this section, we will give an example to illustrate our main results.

Example 1. Consider the following fractional p-Laplacian equation:

$$
\begin{aligned}
& D_{0^{+}}^{3 / 4} \phi_{3}\left(D_{0^{+}}^{1 / 2} x(t)\right) \\
& \quad=-\frac{49}{6}+\frac{1}{6} x^{2}(t)+t e^{-\left|D_{0^{+}}^{1 / 2} x(t)\right|}, \quad t \in[0,1] .
\end{aligned}
$$

Corresponding to (5), we get that $p=3, \alpha=1 / 2, \beta=3 / 4$, and

$$
f(t, u, v)=-\frac{49}{6}+\frac{1}{6} u^{2}+t e^{-|v|} .
$$

Choose $a(t)=10, b(t)=1 / 6$, and $c(t)=0$. By a simple calculation, we can obtain that $\|b\|_{\infty}=1 / 6,\|c\|_{\infty}=0$ and

$$
\frac{2}{\Gamma(3 / 4+1)}\left(\frac{1 / 6}{(\Gamma(1 / 2+1))^{2}}+0\right)<1 .
$$

Obviously, (42) subject to boundary value conditions (6) (or (7)) satisfies all assumptions of Theorem 7 (or Theorem 8). Hence, FBVP (42) and (6) (or FBVP (42) and (7)) has at least one solution.

\section{Acknowledgments}

This work was supported by the Fundamental Research Funds for the Central Universities (2012QNA50) and the National Natural Science Foundation of China (11271364).

\section{References}

[1] R. Metzler and J. Klafter, "Boundary value problems for fractional diffusion equations," Physica A, vol. 278, no. 1-2, pp. 107$125,2000$.

[2] H. Scher and E. W. Montroll, "Anomalous transit-time dispersion in amorphous solids," Physical Review B, vol. 12, no. 6, pp. 2455-2477, 1975.

[3] F. Mainardi, "Fractional diffusive waves in viscoelastic solids," in Nonlinear Waves in Solids, J. L. Wegner and F. R. Norwood, Eds., Fairfield, 1995.

[4] K. Diethelm and A. D. Freed, "On the solution of nonlinear fractional order differential equations used in the modeling of viscoplasticity," in Scientific Computing in Chemical Engineering II: Computational Fluid Dynamics, Reaction Engineering and Molecular Properties, F. Keil, W. Mackens, H. Voss, and J. Werther, Eds., Springer, Heidelberg, Germany, 1999. 
[5] L. Gaul, P. Klein, and S. Kemple, "Damping description involving fractional operators," Mechanical Systems and Signal Processing, vol. 5, no. 2, pp. 81-88, 1991.

[6] W. G. Glockle and T. F. Nonnenmacher, "A fractional calculus approach to self-similar protein dynamics," Biophysical Journal, vol. 68 , pp. 46-53, 1995.

[7] F. Mainardi, "Fractional calculus: some basic problems in continuum and statistical mechanics," in Fractals and Fractional Calculus in Continuum Mechanics, A. Carpinteri and F. Mainardi, Eds., vol. 378 of CISM Courses and Lectures, pp. 291348, Springer, New York, NY, USA, 1997.

[8] R. Metzler, W. Schick, H. G. Kilian, and T. F. Nonnenmacher, "Relaxation in filled polymers: a fractional calculus approach," Journal of Chemical Physics, vol. 103, no. 16, pp. 7180-7186, 1995.

[9] E. Pereira, C. A. Monje, B. M. Vinagre, and F. Gordillho, "Matlab toolbox for the analysis of fractional order systems with hard nonlinearities," in Proceedings of the First IFAC Workshop on Fractional Differentiation and Its Applications (FDA '04), pp. 214-219, Bordeaux, France, July 2004.

[10] A. Babakhani and V. Daftardar-Gejji, "Existence of positive solutions of nonlinear fractional differential equations," Journal of Mathematical Analysis and Applications, vol. 278, no. 2, pp. 434-442, 2003.

[11] D. Delbosco and L. Rodino, "Existence and uniqueness for a nonlinear fractional differential equation," Journal of Mathematical Analysis and Applications, vol. 204, no. 2, pp. 609-625, 1996.

[12] A. A. Kilbas and J. J. Trujillo, "Differential equations of fractional order: methods, results and problems-I," Applicable Analysis, vol. 78, no. 1-2, pp. 153-192, 2001.

[13] A. A. Kilbas and J. J. Trujillo, "Differential equations of fractional order: methods, results and problems-II," Applicable Analysis, vol. 81, no. 2, pp. 435-493, 2002.

[14] R. P. Agarwal, D. O’Regan, and S. Staněk, "Positive solutions for Dirichlet problems of singular nonlinear fractional differential equations," Journal of Mathematical Analysis and Applications, vol. 371, no. 1, pp. 57-68, 2010.

[15] Z. Bai, "On solutions of some fractional $m$-point boundary value problems at resonance," Electronic Journal of Qualitative Theory of Differential Equations, vol. 37, pp. 1-15, 2010.

[16] M. Benchohra, S. Hamani, and S. K. Ntouyas, "Boundary value problems for differential equations with fractional order and nonlocal conditions," Nonlinear Analysis: Theory, Methods \& Applications, vol. 71, no. 7-8, pp. 2391-2396, 2009.

[17] Z. Bai and H. Lü, "Positive solutions for boundary value problem of nonlinear fractional differential equation," Journal of Mathematical Analysis and Applications, vol. 311, no. 2, pp. 495-505, 2005.

[18] M. El-Shahed and J. J. Nieto, "Nontrivial solutions for a nonlinear multi-point boundary value problem of fractional order," Computers \& Mathematics with Applications, vol. 59, no. 11, pp. 3438-3443, 2010.

[19] W. Jiang, "The existence of solutions to boundary value problems of fractional differential equations at resonance," Nonlinear Analysis: Theory, Methods \& Applications, vol. 74, no. 5, pp. 1987-1994, 2011.

[20] N. Kosmatov, "A boundary value problem of fractional order at resonance," Electronic Journal of Differential Equations, no. 135, pp. 1-10, 2010.

[21] X. Su, "Boundary value problem for a coupled system of nonlinear fractional differential equations," Applied Mathematics Letters, vol. 22, no. 1, pp. 64-69, 2009.
[22] L. S. Leibenson, "General problem of the movement of a compressible fluid in a porous medium," Izvestiia Akademii Nauk Kirgizskoĭ SSSR, pp. 7-10, 1983 (Russian).

[23] T. Chen, W. Liu, and C. Yang, "Antiperiodic solutions for Liénard-type differential equation with $p$-Laplacian operator," Boundary Value Problems, vol. 2010, Article ID 194824, 12 pages, 2010.

[24] D. Jiang and W. Gao, "Upper and lower solution method and a singular boundary value problem for the one-dimensional $p$ Laplacian," Journal of Mathematical Analysis and Applications, vol. 252, no. 2, pp. 631-648, 2000.

[25] L. F. Lian and W. G. Ge, "The existence of solutions of $m$ point $p$-Laplacian boundary value problems at resonance," Acta Mathematicae Applicatae Sinica, vol. 28, no. 2, pp. 288-295, 2005.

[26] B. Liu and J. S. Yu, "Existence of solutions for the periodic boundary value problems with $p$-Laplacian operator," Journal of Systems Science and Mathematical Sciences, vol. 23, no. 1, pp. 76-85, 2003.

[27] J. J. Zhang, W. B. Liu, J. B. Ni, and T. Y. Chen, "Multiple periodic solutions of $p$-Laplacian equation with one-side Nagumo condition," Journal of the Korean Mathematical Society, vol. 45, no. 6, pp. 1549-1559, 2008.

[28] T. Chen and W. Liu, "An anti-periodic boundary value problem for the fractional differential equation with a $p$-Laplacian operator," Applied Mathematics Letters, vol. 25, no. 11, pp. 16711675, 2012.

[29] S. Yao, G. Wang, Z. Li, and L. Yu, "Positive solutions for three-point boundary value problem of fractional differential equation with $p$-Laplacian operator," Discrete Dynamics in Nature and Society, vol. 2013, Article ID 376938, 7 pages, 2013.

[30] K. S. Miller and B. Ross, An Introduction to the Fractional Calculus and Fractional Differential Equations, John Wiley \& Sons, New York, NY, USA, 1993.

[31] I. Podlubny, Fractional Differential Equations, vol. 198 of Mathematics in Science and Engineering, Academic Press, San Diego, Calif, USA, 1999.

[32] S. G. Samko, A. A. Kilbas, and O. I. Marichev, Fractional Integrals and Derivatives: Theory and Applications, Gordon and Breach Science, Yverdon, Switzerland, 1993.

[33] A. A. Kilbas, H. M. Srivastava, and J. J. Trujillo, Theory and Applications of Fractional Differential Equations, Elsevier, Amsterdam, The Netherlands, 2006.

[34] R. Manásevich and J. Mawhin, "Periodic solutions for nonlinear systems with $p$-Laplacian-like operators," Journal of Differential Equations, vol. 145, no. 2, pp. 367-393, 1998. 


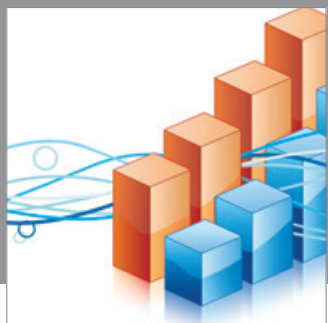

Advances in

Operations Research

mansans

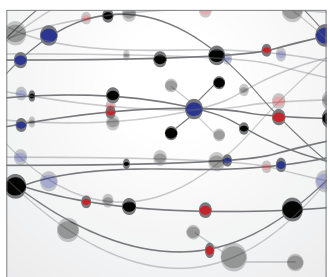

The Scientific World Journal
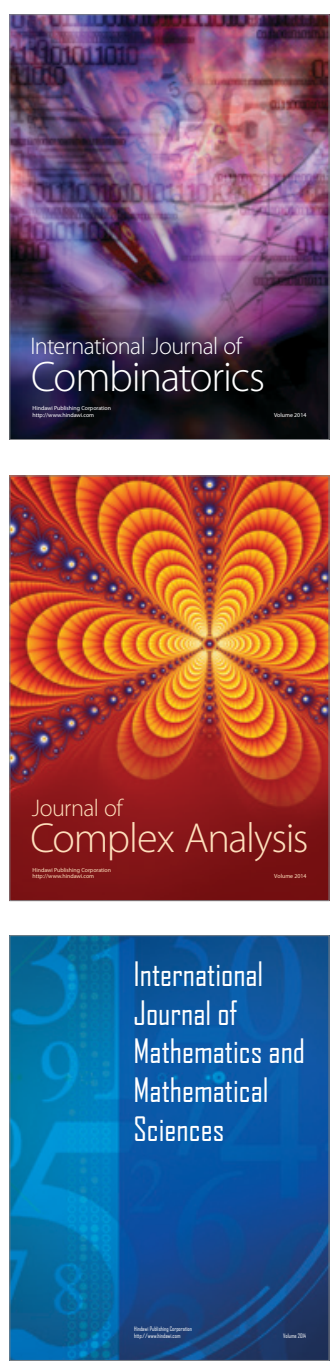
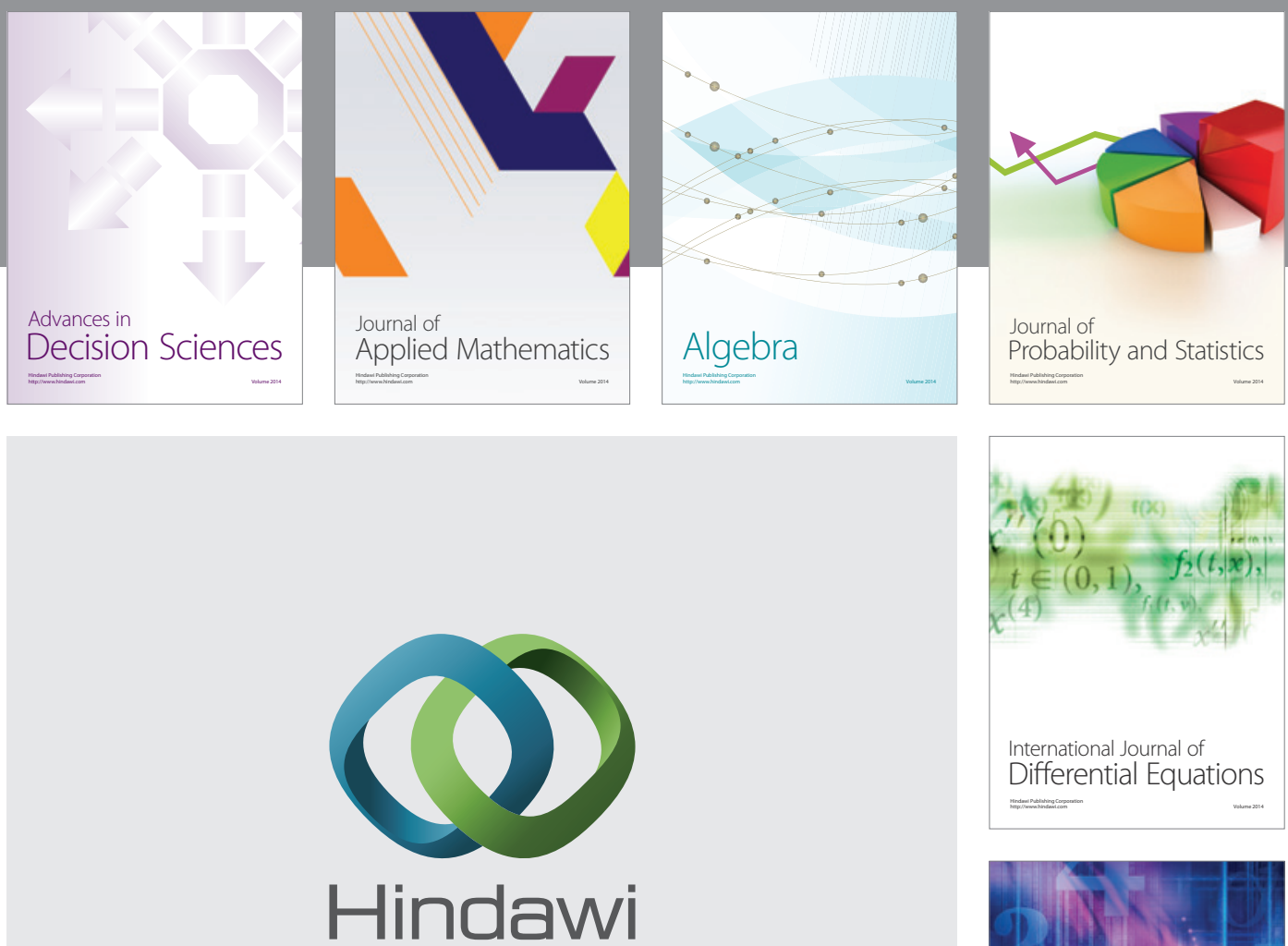

Submit your manuscripts at http://www.hindawi.com
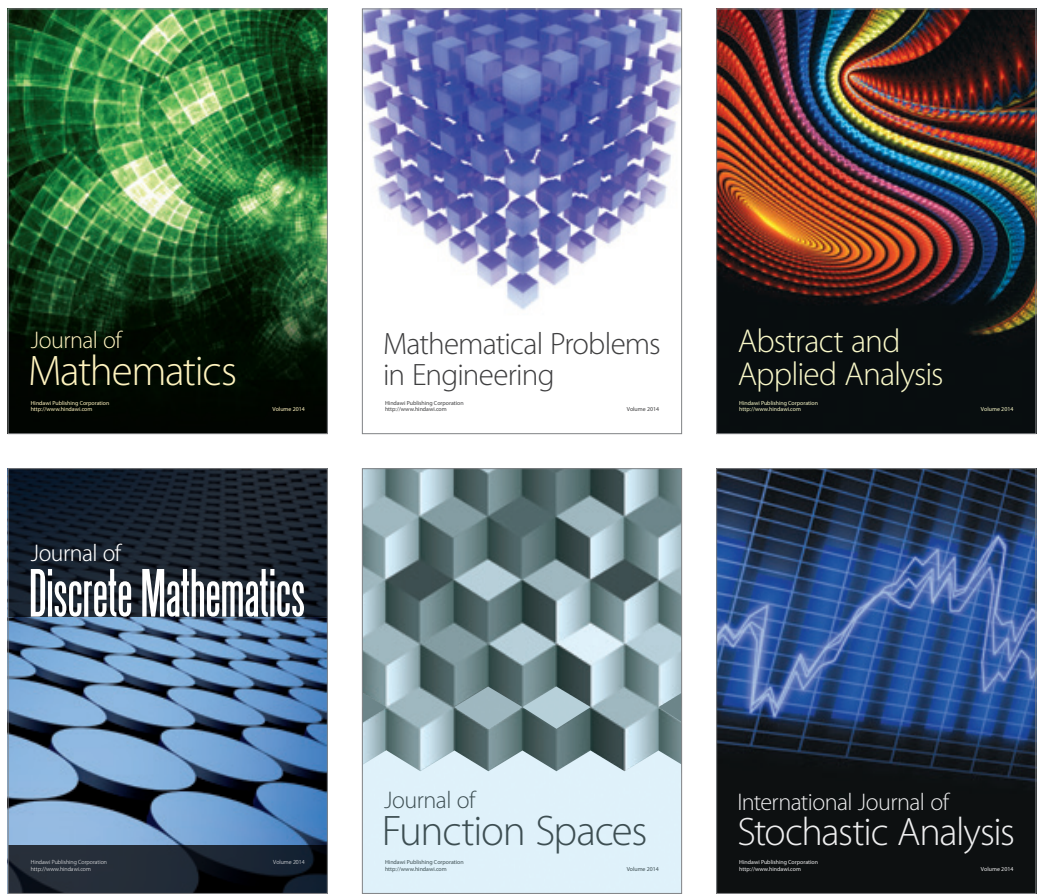

Journal of

Function Spaces

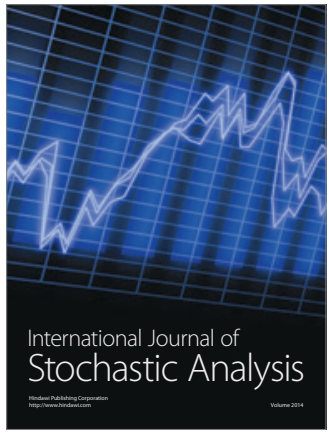

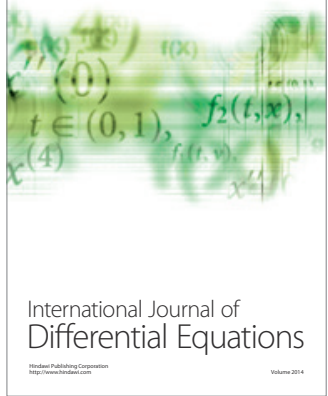
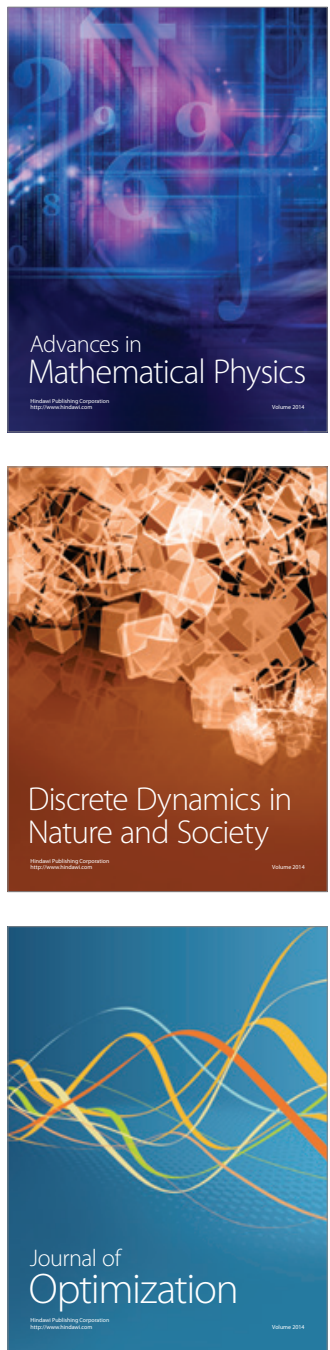